\title{
Curcumin protects neuronal cells against status epilepticus- induced hippocampal damage through induction of autophagy and inhibition of necroptosis
}

\begin{tabular}{|r|l|}
\hline Journal: & Canadian Journal of Physiology and Pharmacology \\
\hline Manuscript ID & cjpp-2016-0154.R2 \\
\hline Danuscript Type: & Article \\
\hline Complete List of Authors: & $\begin{array}{l}\text { Wang, Jin; School of pharmaceutical Science, Central South University } \\
\text { Liu, Yuan; Xiangya School of Medicine, Central South University } \\
\text { Li, Xiao-Hui; School of pharmaceutical Science, Central South University } \\
\text { Zeng, Xiang-Chang; Xiangya Hospital, Central South University; Hunan } \\
\text { Key Laboratory of Pharmacogenetics, Central South University } \\
\text { Li, Jian; Xiangya School of Medicine, Central South University, } \\
\text { Zhou, Jun; Xiangya Hospital, Central South University } \\
\text { Xiao, Bo; Xiangya Hospital, Central South University } \\
\text { Hu, Kai; Xiangya Hospital, Central South University }\end{array}$ \\
\hline Keyword: & \begin{tabular}{l} 
status epilepticus, hippocampus, autophagy, necroptosis, curcumin \\
\hline
\end{tabular} \\
\hline
\end{tabular}




\section{Curcumin protects neuronal cells against}

\section{status epilepticus-induced hippocampal damage through induction of autophagy and inhibition of necroptosis}

Jin Wang ${ }^{\text {b,1 }}$, Yuan Liu ${ }^{\mathrm{c}, 1}$, Xiao- Hui Li ${ }^{\mathrm{b}}$, Xiang-Chang Zeng ${ }^{\mathrm{d}, \mathrm{e}}$, Jian $\mathrm{Li}^{\mathrm{f}}$, Jun Zhou ${ }^{\mathrm{g}}$, Bo Xiao ${ }^{\mathrm{a}}$, and Kai Hu ${ }^{\mathrm{a}}$

${ }^{a}$ Department of Neurology, Xiangya Hospital, Central South University, Changsha 410008, China.

${ }^{b}$ Department of Pharmacology, School of pharmaceutical Science, Central South University, Changsha 410000, China.

${ }^{c}$ Department of Anesthesia, Xiangya School of Medicine, Central South University, Changsha 410008,China.

${ }^{\mathrm{d}}$ Department of Clinical Pharmacology, Xiangya Hospital, Central South University, Changsha 410008,China.

${ }^{\mathrm{e}}$ Institute of Clinical Pharmacology, Hunan Key Laboratory of Pharmacogenetics, Central South University, Changsha 410078, China.

${ }^{\mathrm{f}}$ Department of Laboratory Medicine, Xiangya School of Medicine, Central South University, Changsha 410008, China.

${ }^{\mathrm{g}}$ Medical Science Research Center, Xiangya Hospital, Central South University, Changsha 410008, China.

Corresponding author: Kai $\mathrm{Hu}$

Department of Neurology, Xiangya Hospital, Central South University, 87 Xiangya Road, Changsha, Hunan, P. R. China, 410008, E-mail: kaihu716@126.com, Phone: 86+13187097846.

\footnotetext{
${ }^{1}$ These authors contributed equally to this work.
} 


\begin{abstract}
Status epilepticus, the most severe form of epilepsy, is characterized by progressive functional and structural damage in the hippocampus, ultimately leading to the development and clinical appearance of spontaneous, recurrent seizures. Though the pathogenesis underlying epileptogenesis processes remains unclear, a substantial body of evidence has shown that status epilepticus acts as an important initial factor in triggering epileptogenesis. Notably, besides classical cell death mechanisms such as apoptosis and necrosis, two novel regulators of cell fate known as necroptosis and autophagy, are demonstrated to be involved in neuronal damage in various neurodegenerative and neuropsychiatric disorders. However, whether necroptosis and autophagy play a role in post-status epilepticus rat hippocampus and other epilepsy mechanisms deserves further research effort. In addition, research is needed to determine whether compounds from traditional Chinese herbs possess anti-epileptic effects through the modulation of necroptosis and autophagy. In this study, we found that curcumin, a polyphenolic phytochemical extracted from the Curcuma longa plant, protects neuronal cells against status epilepticus-induced hippocampal neuronal damage in the lithium-pilocarpine-induced status epilepticus rat model through induction of autophagy and inhibition of necroptosis.
\end{abstract}

\title{
Keywords
}

Curcumin; status epilepticus; hippocampus; autophagy; necroptosis 


\section{Introduction}

Epilepsy is one of the world's most common neurological disorders, and most evidence has supported status epilepticus (SE) as an important initial factor in triggering epileptogenesis. Even though the detailed pathogenesis remains unclear, a variety of evidence has indicated that hippocampal neuronal damage, including apoptosis and necroptosis, participates in epileptogenesis (Cheung et al. 2005; Dingledine et al. 2014; Murphy et al. 2007). However, recent findings have also provided novel insights into two other mechanisms associated with cellular death, namely autophagy and necroptosis, and their role in modulating the fate of neuronal cells (Hung et al. 2015; Vieira et al. 2014; Xu and Zhang 2011).

In general, autophagy is a catabolic process that plays a vital role in cellular homeostasis by mediating the turnover of cytoplasmic constituents (McMahon et al., 2012). In the autophagy process, it is widely recognized that the Beclin family member, Beclin-1, and the conversion of the nonlipidated-form of microtubule-associated protein 1 light chain 3 (LC3-I) to the phosphatidylethanolamine-conjugated form (LC3-II) are required. Up to now, several studies have indicated the involvement of autophagy impairment in progressive myoclonus epilepsies (e.g. Lafora disease), but detailed mechanisms of autophagy in epilepsy are unclear (Duran et al. 2014; McMahon et al. 2012; Polajnar and Zerovnik 2011; Puri and Ganesh 2012).

Necroptosis is commonly considered a back-up cell death pathway, which often is recruited when apoptosis is inhibited (Murphy and Silke 2014). In particular, apoptosis occurs during caspase- 8 activation, but if caspase- 8 is inhibited, both receptor interaction protein kinase-1 and -3 (RIP-1 and RIP-3) as well as the mixed lineage kinase domain-like (MLKL) protein are phosphorylated before activation of the necrosome and induction of necroptosis (Linkermann and Green 2014; Nikoletopoulou et al. 2013; WuLiu and Li 2012). Research evidence has indicated that novel mechanisms of cell death, including autophagy, phagoptosis, necroptosis, and pyroptosis, are likely all responsible for epileptogenesis (BollinoBalan and 
Aurelian 2015; Dingledine et al. 2014).

Curcumin, a biologically active natural compound (diferuoyl methane) acquired from the rhizomes of turmeric, is one of the precious chemical substances reported to possess pro-autophagy or anti-necroptosis efficacies (Akkoc et al. 2015; Buyuklu et al. 2014; Guan et al. 2016; Guo et al. 2016; McMahon et al. 2012; Seo et al. 2016; Xiao et al. 2013). Curcumin hormesis may also mediate the cross-talk between autophagy and neuronal cell death in nerve disease (Duran et al. 2014; Rainey et al. 2015). Recent studies have suggested that curcumin can exert protective effects on epilepsy in animal models, but these findings mainly focus on anti-inflammatory and antioxidant aspects of its effects in modulating epileptogenesis, and little is known about the possible connection of its anti-epilepsy efficacy with modulation of autophagy and necroptosis (Ahmad 2013; DU et al. 2012; Jiang et al. 2015; Kaur et al. 2015).

This study aimed to assess alteration of autophagy and necroptosis together with the status of neuronal survival or death in epilepsy using the lithium-pilocarpine-induced SE rat model. In particular, we also explored the neuroprotective effects of curcumin against SE-induced hippocampal neuronal cell death through induction of autophagy proteins (Beclin-1 and LC3) and inhibition of necroptosis proteins (MLKL and RIP-1). 


\section{Methods}

\section{The rat SE model and curcumin treatment}

Male Sprague-Dawley (SD) rats (6-8 weeks of age, weighing 220-250 g, from the Animal Unit of Central South University, P. R. China) were used. All SD rats were housed in a room with controlled temperature $\left(18{ }^{\circ} \mathrm{C}-25^{\circ} \mathrm{C}\right)$ and humidity $(50 \%-60 \%)$ and an alternating 12-h light-dark cycle. Food and water were available ad libitum. All experiments were performed according to Guidelines of the National Institutes of Health on Use and Care of Laboratory Animals. The Animal Care and Use Committee at Xiangya Medical College of Central South University approved the study protocol.

The rat SE model was generated via injection of lithium-pilocarpine as previously described (Hu et al. 2012). Briefly, all animals were randomly divided into four groups: the control, model, curcumin (low-dose), and curcumin (high-dose) groups. In the model group ( $n=6)$, lithium chloride $(125 \mathrm{mg} / \mathrm{kg}$, i.p., Sigma, USA) was administered 18-20 hours prior to the injection of pilocarpine $(20 \mathrm{mg} / \mathrm{kg}$, i.p., Sigma) in order to induce SE. In the curcumin-treated SE groups ( $n=6$ for low-dose and $n=6$ for high-dose), before SE induction, rats were administered curcumin at a dose of 200 $\mathrm{mg} / \mathrm{kg} /$ day or $300 \mathrm{mg} / \mathrm{kg} /$ day via gavage for 2 weeks respectively. Rats in the control group $(n=6)$ were treated with normal saline. All rats were housed in the same environment. Continuous observation of animal behavior was performed after the successful establishment of the SE model. All rats were injected with $10 \%$ chloral hydrate (3 $\mathrm{mL} / \mathrm{kg}$, i.p.) for anesthetization. After decapitation, hippocampi were quickly removed from the brain at $4 h, 24 h$, and $72 h$ post-SE, over ice-cold surfaces and frozen in liquid nitrogen. Animals for morphology research were perfused with saline and $4 \%$ paraformaldehyde.

\section{Western blot analysis}

The western blotting analysis was performed as previously described (Hu et al., 2012). Hippocampal tissue samples were homogenized in lysis buffer, and lysates were separated using $10 \%$ or $12 \%$ sodium dodecyl sulfate polyacrylamide gel 
electrophoresis (SDS-PAGE) and then transferred to polyvinylidene fluoride (PVDF) membranes using the Bio-Rad system (Bio-Rad, USA). For studies of autophagy and necroptosis proteins, including LC3B, Beclin-1, RIP, and MLKL, blots were incubated with the following primary antibodies: $\beta$-actin (1:2000, Sigma-Aldrich, USA); LC3B (1:3000, Abcam, UK); Beclin-1 (1:1000, Abcam); RIP-1 (1:1000, Abcam); and MLKL (1:1000, Abcam). Membranes were incubated with relative secondary antibodies, and protein bands were visualized using chemiluminescence reagents (KPL, USA). Integrated optical density (IOD) values of various proteins and $\beta$-actin were measured to explore protein expressions according to the ratio of protein $/ \beta$-actin.

\section{Nissl staining and TUNEL assay}

As previously described (Hu et al., 2012), Nissl staining was performed to detect surviving neurons, and the TUNEL assay was carried out to identify seizure-induced neuronal death. In brief, sections were stained with $0.5 \%$ Cresyl violet (w/v) before they were dehydrated and mounted. Only cells possessing integrated neuronal morphology were counted as surviving. TUNEL staining was performed according to the manufacturer's instructions (Roche Co., Germany). TUNEL-positive cells were ones demonstrating blue-purple nucleic staining. There are 6 animals per group in this experiment. We considered 4 sections in each hippocampal sample for the TUNEL assay as well as for immunohistochemistry. For each section, four non-overlapping visual fields in bilateral hippocampal regions (4 regions: CA1, CA3, dentate gyrus $[\mathrm{DG}]$, Hilus $[H])$ were selected $(\times 400)$, and results were captured by microscope (Olympus, Japan) before means were obtained for statistical analysis.

\section{Immunohistochemistry}

Hippocampus paraffin sections were de-waxed in xylene and rehydrated before eliminating endogenous peroxidase activity. After treatment with goat serum reagent for $20 \mathrm{~min}$, sections were exposed to primary antibodies, including a mouse monoclonal antibody for RIP (1:500, Abcam) and a rabbit polyclonal antibody for MLKL (1:200, Abcam). Sections were later incubated with biotinylated secondary 
antibodies (1:200, Boster Co., China) and a streptavidin-biotin peroxidase complex solution (Boster Co.) before processing by the HPIAS-1000 Color Image Analysis System. For each section, four non-overlapping visual fields in bilateral rat hippocampal regions $(\mathrm{CA} 1, \mathrm{CA} 3, \mathrm{DG}, H)$ were selected per high-power field $(\times 400)$, and the RIP- or MLKL-positive particles were analyzed for statistics.

\section{Transmission electron microscopy}

Transmission electron microscopy was used to investigate changes of autophagy in the hippocampus. Briefly, the hippocampal tissues were cut into small pieces $(\sim 2$ $\mathrm{mm})$. After primary fixation of these sections in glutaraldehyde $(2.5 \%)$ prepared in sodium cacodylate buffer $(0.1 M, \mathrm{pH} 7.2)$ for $2 h$, post-fixation was carried out in osmium tetraoxide (1\%) for 1-2 $h$ followed by dehydration and embedding in araldite and DDSA medium. Soon after, tissues were baked at $65{ }^{\circ} \mathrm{C}$ for $48 \mathrm{~h}$. Finally, tissues were cut into thin sections (60-90 nm) by ultramicrotome (Leica EMUC 67) and were stained with uranyl acetate and lead citrate (2\%) for contrast. Examination of hippocampal sections was performed by transmission electron microscope (Tecnai G2 Spirit transmission electron microscope equipped with Gatan CCD/Orius camera at 60 $\mathrm{kV})$.

\section{Statistical analysis}

Statistical analysis was performed using SPSS 18.0 software. All data were analyzed using one-way ANOVA followed by the Newman-Keuls test and are represented as mean $\pm \mathrm{SD}$. For statistical analysis, $p<0.05$ was used to indicate a statistically significant result. 


\section{Results}

Curcumin treatment and neuronal survival and death alterations in post-SE rat hippocampus

Nissl staining and TUNEL assay were used to detect neuronal survival and death in hippocampus in the experiment, respectively. As shown in Fig.1 $(A, F)$ and Fig.2 ( $A$, $F)$, the number of apoptotic neuronal cells was markedly increased whereas the surviving neuronal cells were significantly decreased in post-SE hippocampus when compared with controls; this tendency was similar in almost all four regions of the hippocampus (CA1, CA3, DG, and $H$ ) at both 24-h and 72-h time points, except for the TUNEL result at the 72-h time point, which needs more exploration. Quantification analysis results, including distinct hippocampal regions, also showed a similar tendency (Fig.1 $B-E, G-J$ and Fig.2 $B-E, G-J)$.

However, after pretreatment with curcumin, the number of apoptotic neurons markedly decreased and surviving neurons significantly increased at 24-h and 72-h time points. As shown in Fig.1 $(A, F)$ and Fig.2 $(A, F)$, neuronal survival increased and the number of apoptotic neurons significantly decreased in almost all four regions of post-SE hippocampus (CA1, CA3, DG, and $H$ ) in the curcumin-treated groups when compared with the SE model group. Consistently, the quantification analysis results displayed the same tendency (Fig.1 $B-E$, $G-J$ and Fig.2 $B-E, G-J)$.

\section{Necroptosis expression alterations in post-SE rat hippocampus}

In accordance with the changes of neuronal cell fate as described earlier by the results of the TUNEL assay and Nissl staining, the process of necroptosis also showed an alteration in post-SE rat hippocampus. According to immunohistochemical staining and Western blot results, two important proteins associated with the necroptosis process, MLKL and RIP-1, showed significant changes in expression in post-SE hippocampus and as well as in the curcumin-treated groups. In brief, the expression levels of MLKL and RIP-1 proteins increased significantly at $24 h$ and $72 h$ post-SE when compared with the control group (Fig.3 $A, F, K, P$ and Fig.4 $A$ ). However, the 
upregulation of MLKL and RIP-1 proteins was significantly inhibited in the curcumin-treated groups in a dose-dependent manner; the reduction of expression levels was more remarkable in the high-dose curcumin-treated group (Fig.3 B-E, G-J, $L-O, Q-T$ and Fig.4 $B, C)$.

\section{Autophagy expression alterations in post-SE rat hippocampus}

The expression levels of autophagy proteins in post-SE rat hippocampus were detected by Western blot analysis, and morphological changes of autophagosomes in hippocampus were observed by transmission electron microscopy. As shown in Fig.5, the Beclin-1 and LC3BII/LC3BI expression levels as well as the number of autophagosomes showed changes at $4 h$ and $24 h$ in post-SE rat hippocampus. Briefly, the expression levels of Beclin-1 and LC3BII/LC3BI showed an upregulation (Western blot result not shown) and the number of autophagosomes increased at $4 h$ as shown in Fig.5 C. However, the expression levels of Beclin-1 and LC3BII/LC3BI proteins demonstrated a reduction, and the number of autophagosomes decreased at 24 $h$ post-SE when compared with control (Fig.5 $A, B$ and Fig.5 $C$ ). After treatment with curcumin of different dosages, however, the expression levels of Beclin-1 and LC3BII/LC3BI and the number of autophagosomes increased significantly (Fig.5 $A-C)$. 


\section{Discussion}

Epilepsy, one of the world's most common neurological disorders, is characterized by spontaneously recurring seizures, but even now, its pathogenesis remains poorly understood. Despite the lack of mechanistic understanding, it is generally accepted that persistent seizures could directly lead to neuronal injuries. Moreover, compelling evidence over the past several decades has indicated that autophagy and necroptosis likely participate in the neuronal damage process underlying epilepsy. Recent studies have reported that autophagy is impaired in Lafora disease, a progressive neurodegenerative disorder manifested by myoclonus epilepsy (Knecht et al. 2010; Polajnar and Zerovnik 2011; Puri and Ganesh 2010, 2012), and such an autophagy defect may be closely linked with the mammalian target of rapamycin (mTOR) pathway, which negatively regulates the process of autophagy and may be harmful in excessive amounts in epileptogenesis (CaoLi and Cho 2009; Maiese et al. 2013; McMahon et al. 2012). Intriguingly, a recent study revealed that valproic acid, a histone deacetylase inhibitor used to treat epilepsy and mood disorders, induces neuronal cell death through a novel calpain-dependent necroptosis pathway, hinting that necroptosis may also play a significant role in the intricate pathogenesis of epilepsy (Bollino et al. 2015; Dingledine et al. 2014).

These findings indicate that both autophagy and necroptosis may be involved in seizure-induced neuronal damage. As two significant regulators of cellular fate, the cross-talk between autophagy and necroptosis seems to be rather complicated. What remains to be explained more clearly is whether autophagy together with necroptosis plays a role in neuronal cell injury in the hippocampus post-SE or is involved in further epileptic mechanisms. If so, are there substances or drugs that can protect against SE-induced neuronal injury through the regulation of autophagy and necroptosis? To date, more research on these questions is needed.

Our results have demonstrated an alteration in expression of Beclin-1 and LC3 proteins for autophagy and MLKL and RIP-1 proteins for necroptosis in almost all four regions (CA1, CA3, DG, and $H$ ) of the post-SE rat hippocampus. In line with 
expectations, the changes in the number of apoptotic and surviving neurons were coincident with the alterations of autophagy and necroptosis levels. Briefly, the expression of autophagy proteins, Beclin-1 and LC3, were upregulated at the 4-h time point in rat hippocampus post-SE (Western-blot result not shown), and quickly after this transient increase, their expression levels swiftly went down at the 24-h time point post-SE (Fig.5 $A-C$ ). Consistent with this tendency, a decrease in surviving neurons (Fig.1) and an increase in apoptotic neurons appeared at the 24-h time point post-SE (Fig.2), and transmission electron microscopy also showed that the number of autophagosomes were reduced at $24 h$ after the initial increase at $4 h$ post-SE (Fig.5 C). Contrary to autophagy, the expression of necroptosis proteins, MLKL and RIP-1, showed adverse alterations at relative time points (Fig.2-Fig.4). Our research has thus clearly demonstrated that both autophagy and necroptosis participate in neuronal damage in the hippocampus post-SE and that curcumin indeed has neuroprotective effects against neuronal cell death through multifaceted mechanisms (ZhouBeevers and Huang 2011).

The interaction between autophagy, apoptosis, and necroptosis is quite intricate especially regarding autophagy, which could exert both harmful and beneficial effects in different situations (Gump and Thorburn 2011; Leber and Andrews 2010; Linkermann and Green 2014; Nikoletopoulou et al. 2013). According to our results, it seems that autophagy likely acts as a beneficial factor in response to harmful stimuli (e.g. SE and seizure attacks) and necroptosis and apoptosis seem to be the killers that lead to neuronal death in post-SE rat hippocampus. In addition, our study supports the idea that necroptosis can be considered a back-up method for apoptosis when apoptosis fails, since these two mechanisms cooperate to protect hippocampal neuronal cells in response to various stimuli. Stimulated by lithium-pilocarpine-induced SE, necroptosis is activated to aggravate neuronal cell death following hippocampal neuronal cell apoptosis. At the same time, autophagy acts as a housekeeper to maintain neuronal cell homeostasis. At early stages (about $4 h$ ) post-SE, autophagy could be activated as a self-protection mechanism for hippocampal neurons to counteract apoptosis and necroptosis, since a dynamic 
equilibrium exists between them. However, as the situation continues to exacerbate (about $24 h$ post-SE), the balance between autophagy and necroptosis is ultimately upset, and the self-protective function of neurons in the hippocampus collapses, resulting in a decrease of autophagy. Though the detailed mechanisms describing the participation of autophagy and necroptosis in neuronal death of the post-SE hippocampus is still unknown, our study shows that both are related to lithium-pilocarpine-induced SE, and an extraction from traditional Chinese medicinal herbs, namely curcumin, could protect neurons of hippocampus by modulating autophagy and necroptosis.

Curcumin is a polyphenolic phytochemical extraction from the Curcuma botanical genus (family Zingiberaceae; (Kulkarni and Dhir 2010). Besides its wide range of pharmacological properties, such as antioxidant, anti-inflammatory, anti-amyloid, anticancer, etc., recent studies have revealed its potential neuroprotective activity in cell lines and tissues. For instance, an increasing amount of evidence suggests that curcumin may exert protective effects on epilepsy in several animal models. Our results have clearly demonstrated for the first time that curcumin protects neurons in post-SE rat hippocampus through induction of autophagy and inhibition of necroptosis. These results not only provide us with novel insights into epilepsy mechanisms, but also suggest the potential therapeutic benefit of curcumin for the treatment of epilepsy, which deserves to be addressed by future clinical correlative studies. 


\section{Acknowledgements}

This work was supported by the National Natural Science Foundation of China (No. 81301106 to Kai Hu). 


\section{Conflict of Interest}

The authors declare that there is no conflict of interest. 


\section{References}

Ahmad, M. 2013. Protective effects of curcumin against lithium-pilocarpine induced status epilepticus, cognitive dysfunction and oxidative stress in young rats. Saudi. J. Biol. Sci. 20(2): 155-162. PMID: 23961231

Akkoc, Y., Berrak, O., Arisan, E. D., Obakan, P., Coker-Gurkan, A., and Palavan-Unsal, N. 2015. Inhibition of PI3K signaling triggered apoptotic potential of curcumin which is hindered by Bcl-2 through activation of autophagy in MCF-7 cells. Biomed. Pharmacother. 71: 161-171. PMID: 25960232

Bollino, D., Balan, I., and Aurelian, L. 2015. Valproic acid induces neuronal cell death through a novel calpain-dependent necroptosis pathway. J. Neurochem. 133(2): 174-186. PMID:25581256

Buyuklu, M., Kandemir, F. M., Ozkaraca, M., Set, T., Bakirci, E. M., and Topal, E. 2014. Protective effect of curcumin against contrast induced nephropathy in rat kidney: what is happening to oxidative stress, inflammation, autophagy and apoptosis? Eur. Rev. Med. Pharmacol. Sci. 18(4): 461-470. PMID:24610611

Cao, R., Li, A., and Cho, H. Y. 2009. mTOR signaling in epileptogenesis: too much of a good thing? J. Neurosci. 29(40): 12372-12373. PMID:19812312

Cheung, E. C., Melanson-Drapeau, L., Cregan, S. P., Vanderluit, J. L., Ferguson, K. L., McIntosh, W. C., et al. 2005. Apoptosis-inducing factor is a key factor in neuronal cell death propagated by BAX-dependent and BAX-independent mechanisms. J. Neurosci. 25(6): 1324-1334. PMID:15703386

Dingledine, R., Varvel, N. H., and Dudek, F. E. 2014. When and how do seizures kill neurons, and is cell death relevant to epileptogenesis? Adv. Exp. Med. Biol. 813:109-22. PMID:25012371

DU P, Tang, H. Y., Li, X., Lin, H. J., Peng, W. F., and Ma, Y., et al. 2012. Anticonvulsive and antioxidant effects of curcumin on pilocarpine-induced seizures in rats. Chin. Med. J. (Engl.), 125(11): 1975-1979. PMID:22884064

Duran, J., Gruart, A., Garcia-Rocha, M., Delgado-Garcia, J. M., and Guinovart, J. J. 
2014. Glycogen accumulation underlies neurodegeneration and autophagy impairment in Lafora disease. Hum. Mol. Genet. 23(12): 3147-3156. PMID:24452334

Guan, F., Ding, Y., Zhang, Y., Zhou, Y., Li, M., and Wang, C. 2016. Curcumin Suppresses Proliferation and Migration of MDA-MB-231 Breast Cancer Cells through Autophagy-Dependent Akt Degradation. PLoS One, 11(1): e0146553. PMID:26752181

Gump, J. M., and Thorburn, A. 2011. Autophagy and apoptosis: what is the connection? Trends Cell Biol. 21(7): 387-392. PMID:21561772

Guo, S., Long, M., Li, X., Zhu, S., Zhang, M., and Yang, Z. 2016. Curcumin activates autophagy and attenuates oxidative damage in EA.hy926 cells via the Akt/mTOR pathway. Mol. Med. Rep. 13(3):2187-93. PMID:26781771

Hu, K., Xie, Y. Y., Zhang, C., Ouyang, D. S., Long, H. Y., and Sun, D. N., et al. 2012. MicroRNA expression profile of the hippocampus in a rat model of temporal lobe epilepsy and miR-34a-targeted neuroprotection against hippocampal neurone cell apoptosis post-status epilepticus. BMC Neurosci., 13: 115. PMID:22998082

Hung, S. Y., Huang, W. P., Liou, H. C., and Fu, W. M. 2015. LC3 overexpression reduces Abeta neurotoxicity through increasing alpha7nAchR expression and autophagic activity in neurons and mice. Neuropharmacology, 93: 243-251. PMID:25686800

Jiang, Z., Guo, M., Shi, C., Wang, H., Yao, L., Liu, L., et al. 2015. Protection against cognitive impairment and modification of epileptogenesis with curcumin in a post-status epilepticus model of temporal lobe epilepsy. Neuroscience, 310: 362-371. PMID:26415768

Kaur, H., Patro, I., Tikoo, K., and Sandhir, R. 2015. Curcumin attenuates inflammatory response and cognitive deficits in experimental model of chronic epilepsy. Neurochem. Int. 89: 40-50. PMID:26190183

Knecht, E., Aguado, C., Sarkar, S., Korolchuk, V. I., Criado-Garcia, O., Vernia, S., et al. 2010. Impaired autophagy in Lafora disease. Autophagy, 6(7): 991-993. PMID:20818165

Kulkarni, S. K., and Dhir, A. 2010. An overview of curcumin in neurological 
disorders. Indian. J. Pharm. Sci. 72(2): 149-154. PMID:20838516

Leber, B., and Andrews, D. W. 2010. Closing in on the link between apoptosis and autophagy. F1000 Biol. Rep. 2: 88. PMID:21283600

Linkermann, A., and Green, D. R. 2014. Necroptosis. N. Engl. J. Med. 370(5), 455-465. PMID:24476434

Maiese, K., Chong, Z. Z., Shang, Y. C., and Wang, S. 2013. mTOR: on target for novel therapeutic strategies in the nervous system. Trends Mol. Med. 19(1), 51-60. PMID:23265840

McMahon, J., Huang, X., Yang, J., Komatsu, M., Yue, Z., Qian, J., et al. 2012. Impaired autophagy in neurons after disinhibition of mammalian target of rapamycin and its contribution to epileptogenesis. J. Neurosci. 32(45): 15704-15714. PMID:23136410

Murphy, B., Dunleavy, M., Shinoda, S., Schindler, C., Meller, R., and Bellver-Estelles, C., et al. 2007. Bcl-w protects hippocampus during experimental status epilepticus. Am. J. Pathol. 171(4): 1258-1268. PMID:17702891

Murphy, J. M., and Silke, J. 2014. Ars Moriendi; the art of dying well - new insights into the molecular pathways of necroptotic cell death. EMBO Rep. 15(2): 155-164. PMID:24469330

Nikoletopoulou, V., Markaki, M., Palikaras, K., and Tavernarakis, N. 2013. Crosstalk between apoptosis, necrosis and autophagy. Biochim. Biophys. Act., 1833(12): 3448-3459. PMID:23770045

Polajnar, M., and Zerovnik, E. 2011. Impaired autophagy: a link between neurodegenerative diseases and progressive myoclonus epilepsies. Trends Mol. Med. 17(6): 293-300. PMID:21482188

Puri, R., and Ganesh, S. 2010. Laforin in autophagy: a possible link between carbohydrate and protein in Lafora disease? Autophagy, 6(8): 1229-1231. PMID:20818153

Puri, R., and Ganesh, S. 2012. Autophagy defects in Lafora disease: cause or consequence? Autophagy, 8(2): 289-290. PMID:22301990

Rainey, N., Motte, L., Aggarwal, B. B., and Petit, P. X. 2015. Curcumin hormesis 
mediates a cross-talk between autophagy and cell death. Cell Death Dis. 6: e2003. PMID:26633709

Seo, J. A., Kim, B., Dhanasekaran, D. N., Tsang, B. K., and Song, Y. S. 2016. Curcumin induces apoptosis by inhibiting sarco/endoplasmic reticulum $\mathrm{Ca}(2+)$ ATPase activity in ovarian cancer cells. Cancer Lett. 371(1): 30-37. PMID:26607901 Vieira, M., Fernandes, J., Carreto, L., Anuncibay-Soto, B., Santos, M., Han, J., et al. 2014. Ischemic insults induce necroptotic cell death in hippocampal neurons through the up-regulation of endogenous RIP3. Neurobiol. Dis. 68: 26-36. PMID:24746856 Wu, W., Liu, P., and Li, J. 2012. Necroptosis: an emerging form of programmed cell death. Crit. Rev. Oncol. Hematol. 82(3): 249-258. PMID:21962882

Xiao, K., Jiang, J., Guan, C., Dong, C., Wang, G., Bai, L., et al. 2013. Curcumin induces autophagy via activating the AMPK signaling pathway in lung adenocarcinoma cells. J. Pharmacol. Sci. 123(2): 102-109. PMID:24048094

Xu, M., and Zhang, H. L. 2011. Death and survival of neuronal and astrocytic cells in ischemic brain injury: a role of autophagy. Acta Pharmacol. Sin. 32(9): 1089-1099. PMID:21804578

Zhou, H., Beevers, C. S., and Huang, S. 2011. The targets of curcumin. Curr. Drug Targets, 12(3): 332-347. PMID:20955148 


\section{Figure legends}

Fig.1. Neuronal survival detected in post-SE rat hippocampus at $24 h$ and $72 h$ by Nissl staining. $(\times 400)$

$A$, F: 24-h and 72-h time points for Nissl staining results, respectively. $B-E$, G-J: Quantification of Nissl bodies in rat hippocampal CA1, CA3, dentate gyrus (DG), and hilus $(H)$ regions at 24-h and 72-h time points, respectively.

CTRL: control group; $\quad$ MODEL: epilepsy model group; curcumin $(L)$ : curcumin (low dose) group; curcumin $(H)$ : curcumin (high dose) group. ${ }^{*} p<0.05, * * p<0.01, * * * p<0.001$ versus model group; ${ }^{\#} p<0.05,{ }^{\# \#} p<0.01,{ }^{\# \#} p<0.001$ versus control group ( $n=6$ per group).

Fig.2. Neuronal apoptosis detected in post-SE rat hippocampus at $24 h$ and $72 h$ by TUNEL assay. $(\times 400)$

$A, F$ : 24-h and 72-h time points for TUNEL assay results, respectively. $B-E, G-J$ : Quantification of TUNEL-positive cell units in rat hippocampal CA1, CA3, DG, and $H$ regions at 24-h and 72-h time points, respectively.

CTRL: control group; $\quad$ MODEL: epilepsy model group; curcumin $(L)$ : curcumin (low dose) group; $\operatorname{curcumin}(H)$ : curcumin (high dose) group. $* p<0.05, * * p<0.01, * * * p<0.001$ versus model group; ${ }^{\#} p<0.05,{ }^{\# \#} p<0.01,{ }^{\# \#} p<0.001$ versus control group ( $n=6$ per group).

Fig.3. Expression alterations of MLKL and RIP-1 proteins in post-SE rat hippocampus by immunohistochemistry. $(\times 400)$

$A, F$ : 24-h and 72-h time points for MLKL expression results, respectively.

$K, P: 24-\mathrm{h}$ and 72 -h time points for RIP-1 expression results, respectively. $B-E, G-J$ : Quantification of MLKL-positive immunoreactive products in CA1, CA3, DG, and $H$ regions at 24-h and 72-h time points, respectively. $L-O, Q-T$ : Quantification of RIP-1-positive immunoreactive products in CA1, CA3, 
DG, and $\mathrm{H}$ regions at 24-h and 72-h time points, respectively.

CTRL: control group; $\quad$ MODEL: epilepsy model group;

curcumin $(L)$ : curcumin (low dose) group; $\operatorname{curcumin}(H)$ : curcumin (high dose) group.

${ }^{*} p<0.05,{ }^{*} p p<0.01, * * * p<0.001$ versus model group;

${ }^{\#} p<0.05,{ }^{\# \#} p<0.01,{ }^{\# \#} p<0.001$ versus control group ( $n=6$ per group).

Fig.4. Expression alterations of MLKL and RIP-1 proteins in post-SE rat hippocampus by Western blot analysis.

Western blot analysis of the alterations of MLKL and RIP-1 protein expression in rat hippocampus in the control, model, and curcumin-treated groups (high/low doses) at $24 \mathrm{~h}$ post-SE $(A)$ and the quantification of MLKL $(B)$ and RIP-1 $(C)$ proteins.

CTRL: control group; $\quad$ MODEL: epilepsy model group;

curcumin $(L) / \mathrm{Cu}(L)$ : curcumin (low dose) group;

$\operatorname{curcumin}(H) / \mathrm{Cu}(H)$ : curcumin (high dose) group.

${ }^{*} p<0.05,{ }^{*} p<0.01$ versus control;

${ }^{\#} p<0.05,{ }^{\#} p<0.01$ versus model ( $n=6$ per group).

Fig.5. Western blot analysis of expression alterations of autophagy proteins and transmission electron microscopy for autophagosome morphology.

Western blot analysis of expression levels of autophagy proteins, Beclin-1 $(A)$ and LC3 (B), in rat hippocampus in the control, model, and curcumin-treated groups (high/low doses) at $24 h$ post-SE. Transmission electron microscope images of autophagosomes in rat hippocampus in the control, model, and curcumin-treated groups (high/low doses) at $4 h$ and $24 h$ post-SE $(C)$.

Scale bars: $2 \mu \mathrm{m}$.

CTRL: control group; $\quad$ MODEL: epilepsy model group;

curcumin $(L) / \mathrm{Cu}(L)$ : curcumin (low dose) group;

$\operatorname{curcumin}(H) / \mathrm{Cu}(H)$ : curcumin (high dose) group.

${ }^{*} p<0.05,{ }^{* *} p<0.01$ versus control;

${ }^{\#} p<0.05,{ }^{\#} p<0.01$ versus model ( $n=6$ per group). 

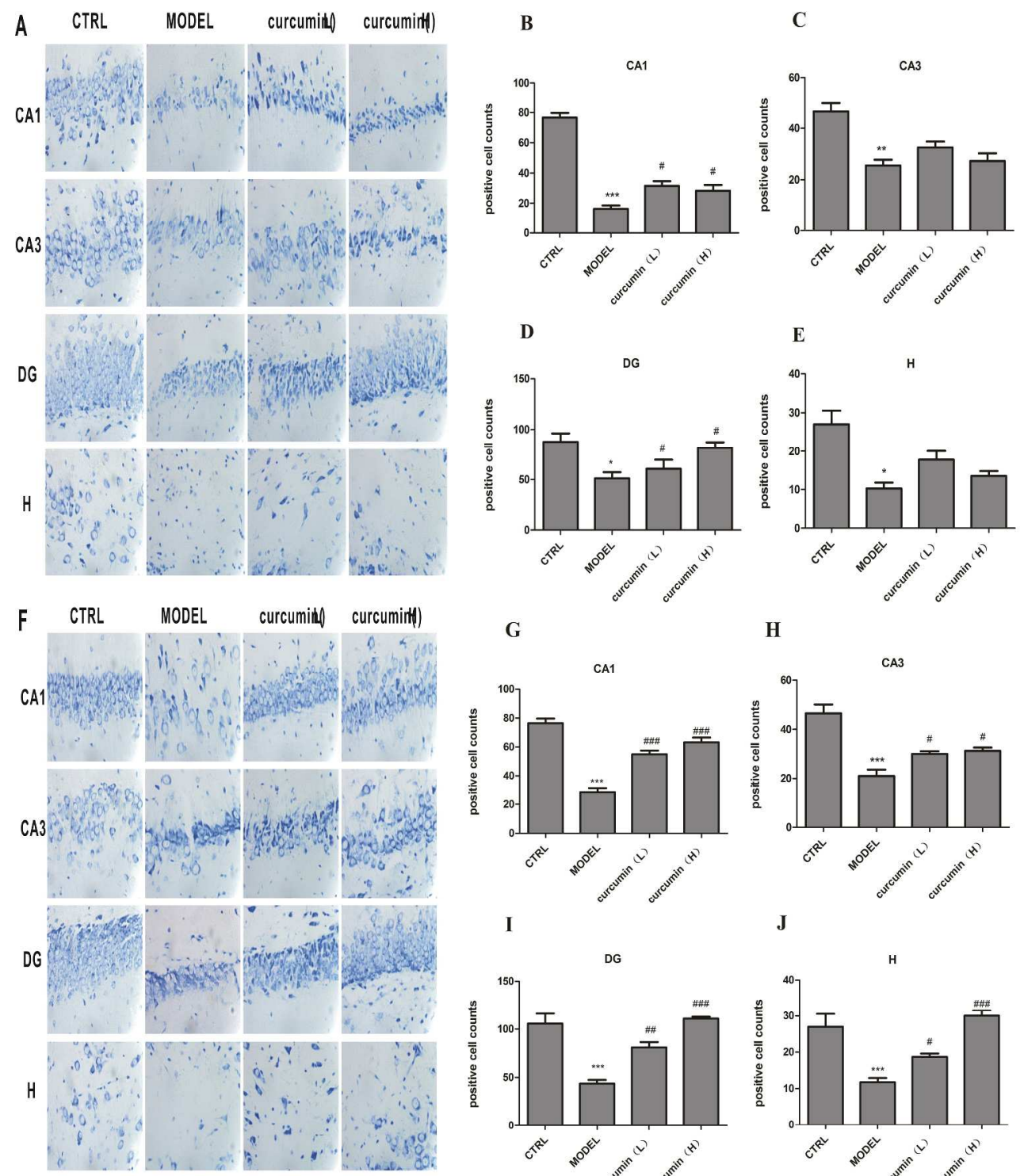

H

CA3
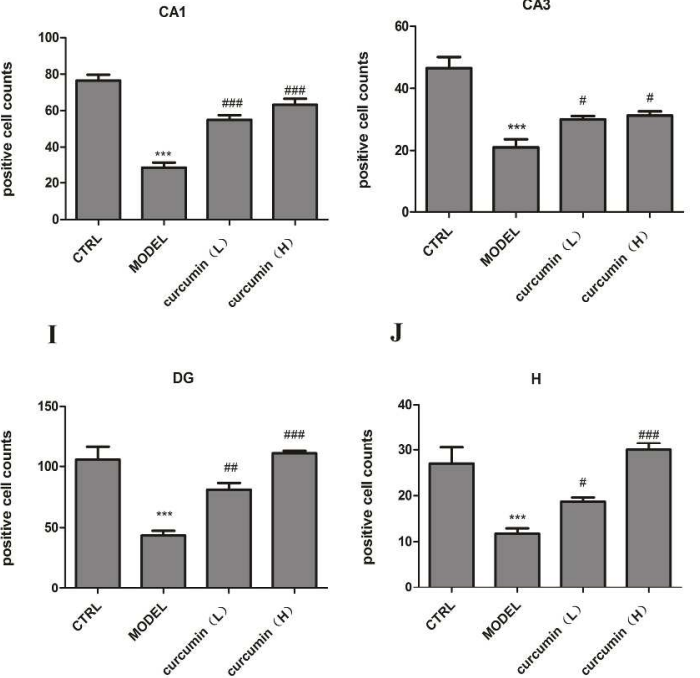

Fig.1. Neuronal survival detected in post-SE rat hippocampus at $24 \mathrm{~h}$ and $72 \mathrm{~h}$ by Nissl staining. $252 \times 308 \mathrm{~mm}(600 \times 600 \mathrm{DPI})$ 

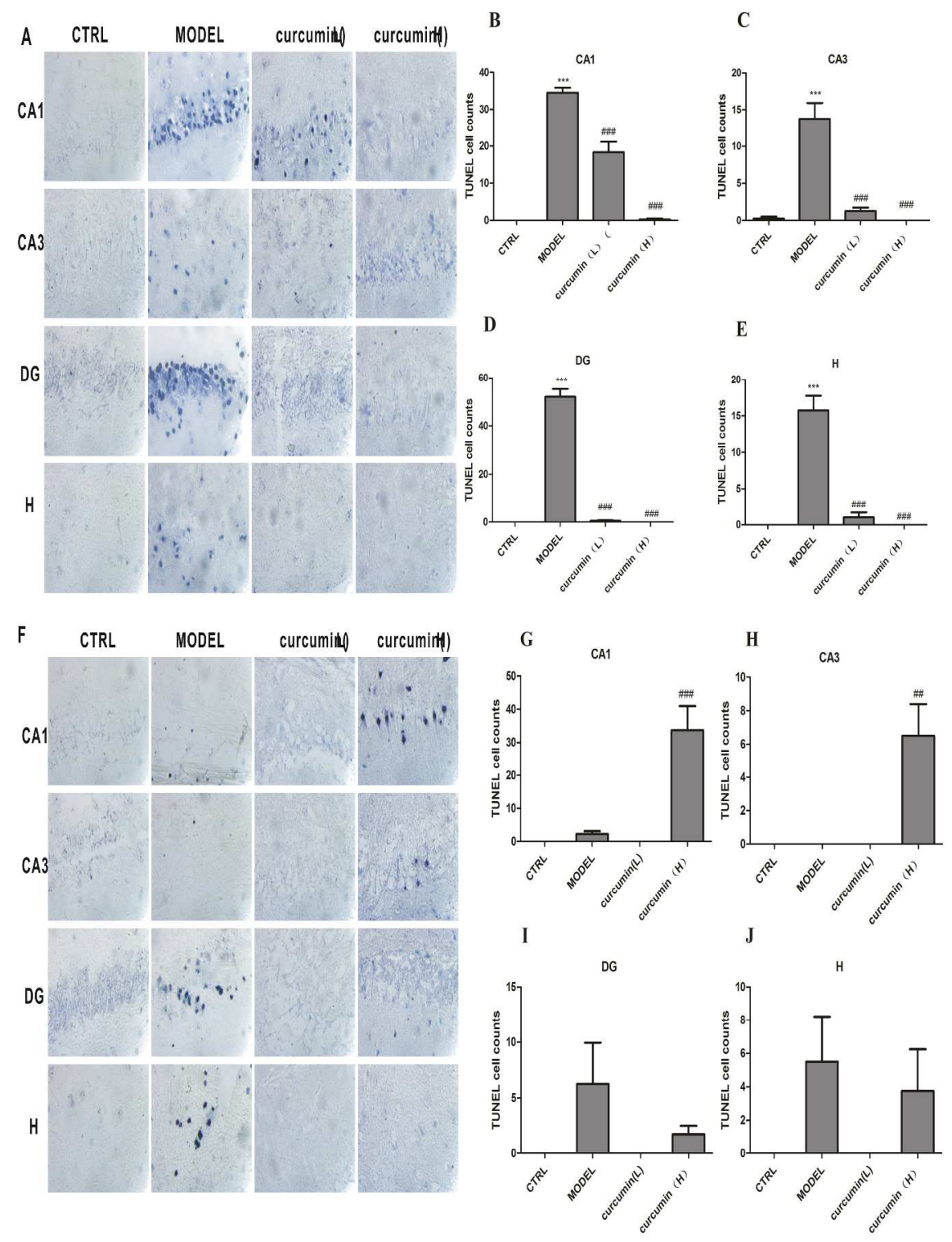

Fig.2. Neuronal apoptosis detected in post-SE rat hippocampus at $24 \mathrm{~h}$ and $72 \mathrm{~h}$ by TUNEL assay. $278 \times 379 \mathrm{~mm}(600 \times 600 \mathrm{DPI})$ 

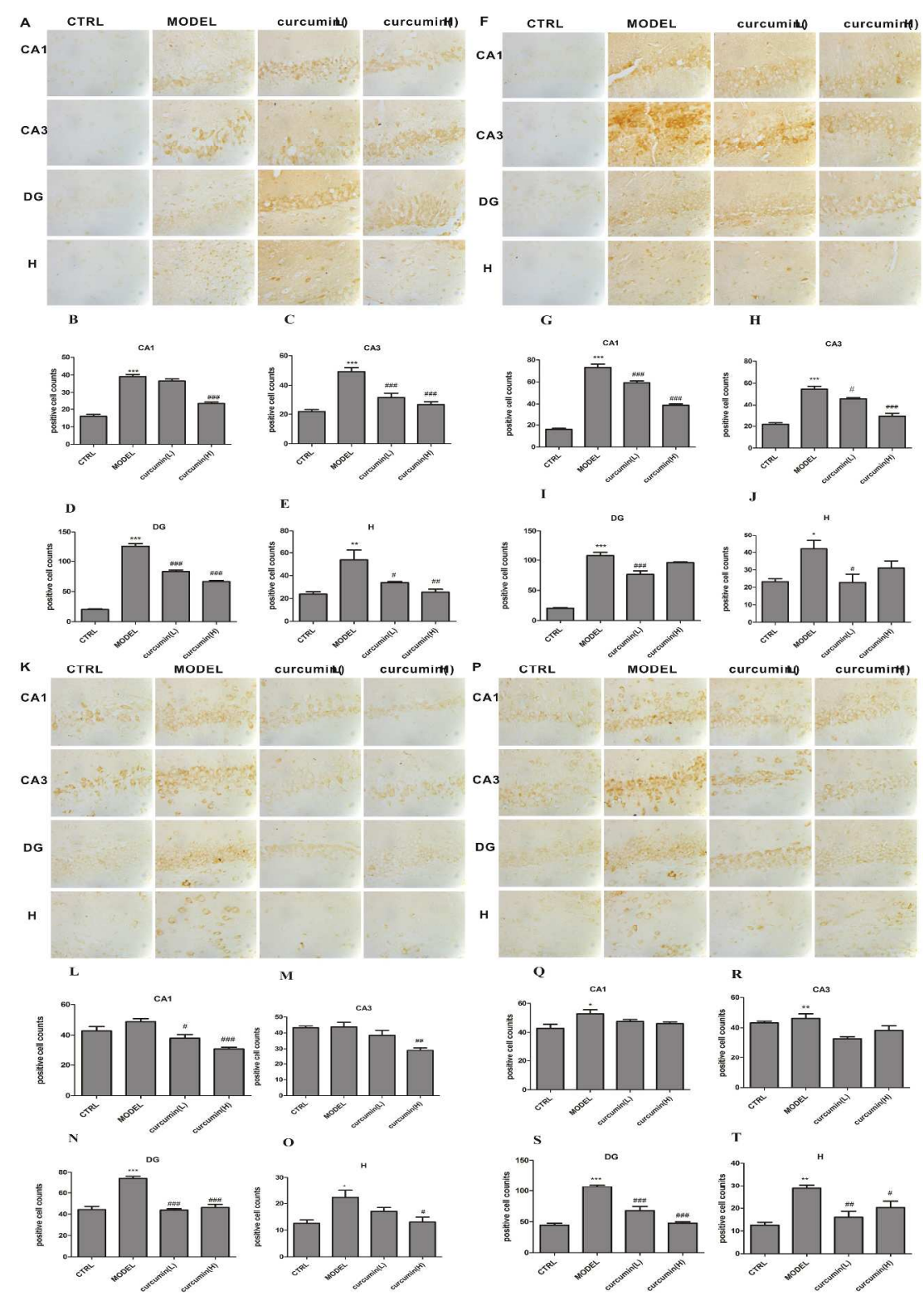

Fig.3. Expression alterations of MLKL and RIP-1 proteins in post-SE rat hippocampus by immunohistochemistry.

$291 \times 417 \mathrm{~mm}(600 \times 600 \mathrm{DPI})$ 
A
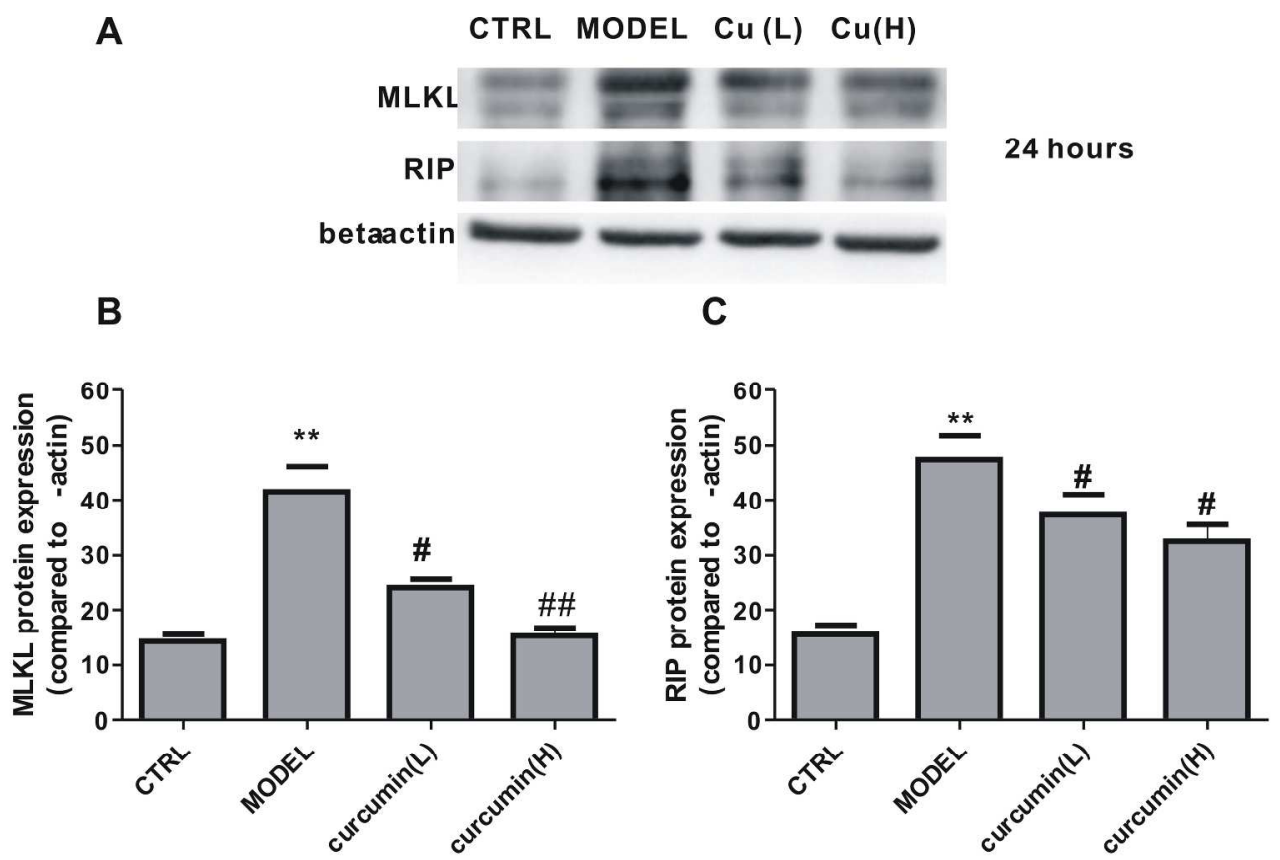

Fig.4. Expression alterations of MLKL and RIP-1 proteins in post-SE rat hippocampus by Western blot analysis.

$129 \times 85 \mathrm{~mm}(600 \times 600 \mathrm{DPI})$ 


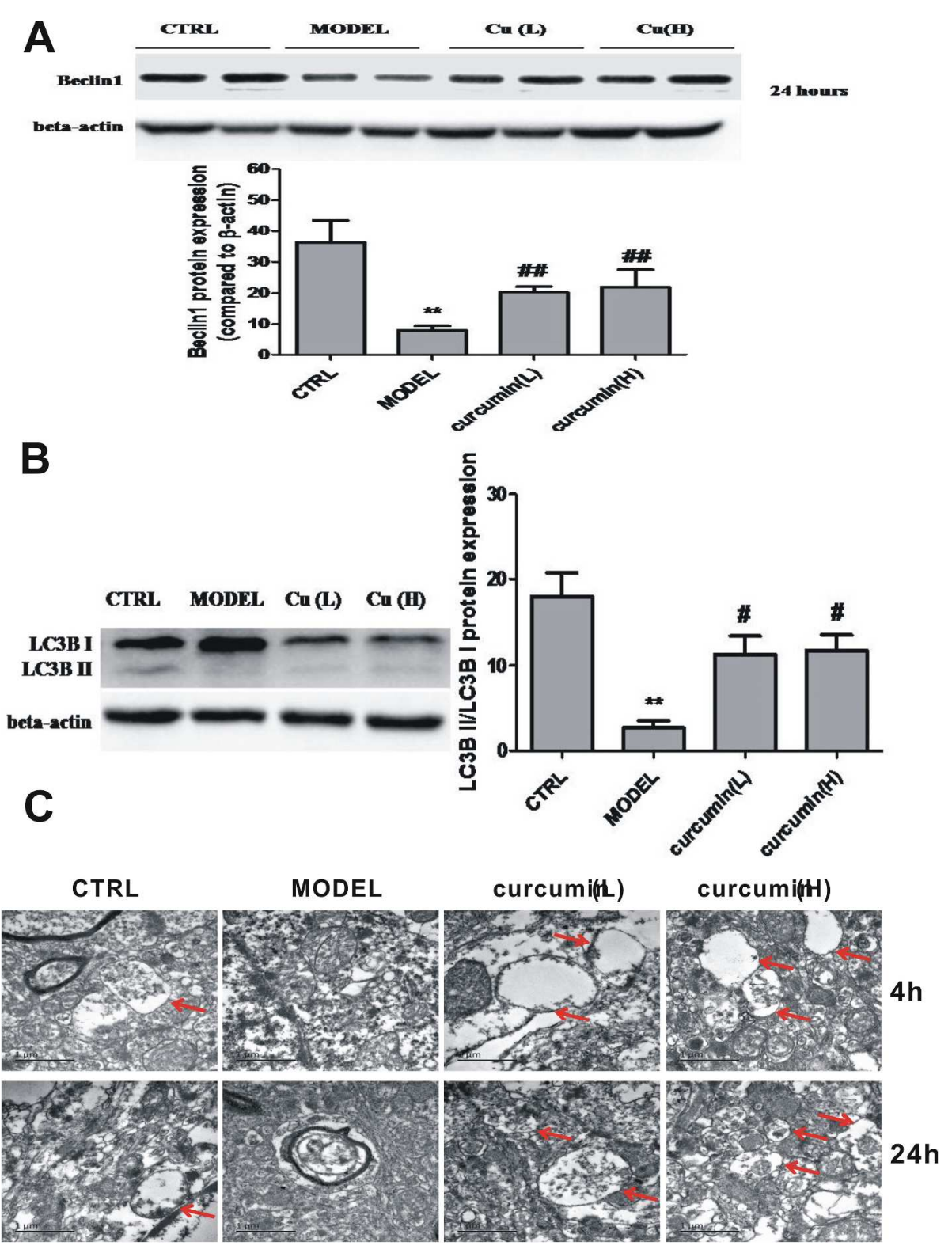

Fig.5. Western blot analysis of expression alterations of autophagy proteins and transmission electron microscopy for autophagosome morphology.

$267 \times 352 \mathrm{~mm}(300 \times 300 \mathrm{DPI})$ 